\title{
Speeding-up exact and fast FIFO-based cache configuration simulation
}

\author{
Masashi Tawada $^{1 \mathrm{a})}$, Masao Yanagisawa ${ }^{2}$, and Nozomu Togawa ${ }^{1 \mathrm{~b})}$ \\ ${ }^{1}$ Dept. of Computer Science and Engineering, Waseda University \\ ${ }^{2}$ Dept. of Electronic and Photonic Systems, Waseda University \\ 3-4-1 Okubo, Shinjuku, Tokyo 169-8555, Japan \\ a) tawada@togawa.cs.waseda.ac.jp \\ b) togawa@togawa.cs.waseda.ac.jp
}

\begin{abstract}
The number of sets, block size, and associativity determine processor's cache configurations. Particularly in embedded systems, their cache configuration can be optimized since their target applications are much limited. Recently, the CRCB method has been proposed for LRU-based (Least Recently Used-based) cache configuration simulation, which can calculate cache hit/miss counts accurately and very fast changing the three parameters. However many recent processors use FIFO-based (First-In-First-Out-based) caches instead of LRU-based caches due to the viewpoints of their hardware costs. In this paper, we propose a speeding-up cache configuration simulation method for embedded applications that uses FIFO as a cache replacement policy. We first prove several properties for FIFO-based caches and then propose a simulation method that can process two or more FIFO-based cache configurations with different cache associativities simultaneously. Experimental results show that our proposed method can obtain accurate cache hits/misses and runs up to $32 \%$ faster than the conventional simulators.
\end{abstract}

Keywords: FIFO, cache simulation, cache memory, cache configuration optimization, embedded systems

Classification: Electron devices, circuits, and systems

\section{References}

[1] T. Austin, E. Larson, and D. Erunst, "SimpleScalar: an infrastructure for computer system modeling," IEEE Trans. Comput., vol. 35, no. 2, pp. 59-67, 2002.

[2] J. Edler and M. D. Hill, "Dinero IV: trace-driven uniprocessor cache simulator," [Online] http://www.cs.wisc.edu/ markhill/DineroIV/.

[3] A. Janapsatya, A. lgnjatovic, and S. Parameswaran, "Finding optimal L1 cache configuration for embedded systems," Proc. ASP-DAC 2006, pp. 796-801, 2006.

[4] C. Lee, M. Potkonjak, and W. H. Mangione-Smith, "MediaBench: a tool for evaluating and synthesizing multimedia and communications systems," Proc. 30th Annual International Symp. Microarchitecture, 1997. 
[5] M. Tawada, M. Yanagisawa, T. Ohtsuki, and N. Togawa, "Exact and fast L1 cache configuration simulation for embedded systems with FIFO/PLRU cache replacement policies," Proc. VLSI-DAT 2011, pp. 247-250, 2011.

[6] N. Tojo, N. Togawa, M. Yanagisawa, and T. Ohtsuki, "Exact and fast L1 cache simulation for embedded systems," Proc. ASP-DAC 2009, pp. 817$822,2009$.

\section{Introduction}

Cache memory access speed cannot increase as expected unless the cache is properly configured. Since application programs running on embedded processors are limited unlike those on general-purpose processors, embedded processors can be optimized for a particular application program. Counting cache hits/misses is the important key to configure such an application-driven cache accordingly.

There are roughly two types of cache hit/miss measuring methods: The first ones just estimate cache hit/miss counts based on cache behavior. The second ones simulate memory accesses and obtain cache hit/miss counts accurately. The first ones run very fast but may have large errors in cache hit/miss counts. The second ones are accurate but can be too much time consuming. This paper targets the second approach, a simulation-based approach $[3,6]$. Simulating cache hits/misses takes too much time and boosting up the cache configuration simulation is the most important key there.

Cache configuration is parameterized by the number of sets, block size, and associativity. Cache configuration simulation counts cache hits/misses on various cache configurations by changing the three parameters from their minimum values to the maximum. Cache configuration is also characterized by its cache replacement policy, which is an algorithm when and where a datum is stored to and discarded from a cache. LRU-based (Least Recently Used-based) and FIFO-based (First-In-First-Out-based) caches are well seen in practical processors. If a processor uses an LRU-based cache whose associativity is too much large, its hardware cost increases too much. On the other hand, FIFO can be implemented with low hardware cost even if its associativity is large. Many practical processors, such as Power4 processors, use FIFO rather than LRU.

This paper proposes an exact and fast L1 instruction/data cache configuration simulation algorithm for embedded applications that use FIFO as a cache replacement policy. Our algorithm is an improved version of the CSG (cache-set-group) algorithm proposed in [5]. In addition to the cache properties proved in [5], we further prove in this paper several properties for FIFO-based caches and propose a boosting up cache configuration simulation strategy. 


\section{Cache configuration simulation method}

\subsection{FIFO-based cache configuration simulation}

We assume FIFO as a cache replacement policy. Each cache set in a cache is represented by a priority queue. In an FIFO-based cache, data in each priority queue are replaced in a first-in-first-out fashion. Cache configuration simulation based on FIFO has a property that priority queues do not have to be updated if cache hit occurs.

Let $s_{0}$ and $b_{0}$ be the minimum number of cache sets and the minimum block size, respectively. Let $s_{m}, b_{m}$, and $a_{m}$ be the maximum number of cache sets, the maximum block size, and the maximum associativity, respectively. Then we explore cache configurations $(s, b, a)$ where

$$
\begin{aligned}
s & =s_{0}, 2 s_{0}, 4 s_{0}, \ldots, s_{m} \\
b & =b_{0}, 2 b_{0}, 4 b_{0}, \ldots, b_{m} \\
a & =1,2,3, \ldots, a_{m}
\end{aligned}
$$

Given a cache configuration $c=(s, b, a)$, let $S(c, i)$ and $S(c, i)_{j}$ be the $i$-th cache set or priority queue on $c$ and the element whose priority is $j$ in $S(c, i)$, where $j=0, \cdots,(a-1)$, respectively. The tag which is the most IN-side in $S(c, i)$ has the priority zero. The tag which is the most OUT-side in $S(c, i)$ has the priority $(a-1)$.

A naive exhaustive algorithm for cache simulation for a particular cache configuration $c=(s, b, a)$ and a particular memory address $A$ is shown in Fig 1 (A). Step 1), Step 3) and Step 4) require a constant time. Step 2) checks one by one a maximum of $a$ elements and then step 2) requires a maximum of $a \mathrm{hit} / \mathrm{miss}$ check counts. We have to repeat Step 1)-Step 4) for all the memory accesses and for all the possible cache configurations.

The CRCB method [6], the very fast cache configuration simulation method, was proposed originally for LRU-based caches, but we proved that the CRCB method can be applied not only to LRU but also to FIFO as well as PLRU (Pseudo-LRU) [5].

\subsection{CSG method [5]}

In [5], we did not only prove that the CRCB method can be applied to FIFO but also we proposed a totally new method, the CSG method, which performs exact and fast FIFO-based cache configuration simulation.

The CSG method groups several priority queues whose behavior is completely the same and just simulates one of them. Now let us focus on two cache configurations $c_{0}=\left(s, b, a_{0}\right)$ and $c_{1}=\left(s, b, a_{1}\right)$, where the number $s$ of cache sets is identical, their block size $b$ is also identical, but their associativity is different $\left(a_{0}<a_{1}\right)$. Let $S\left(c_{0}, i\right)$ and $S\left(c_{1}, i\right)$ be the $i$-th priority queues on $c_{0}$ and $c_{1}$. We assume here that all the tags in $S\left(c_{0}, i\right)$ are included in $S\left(c_{1}, i\right)$ and their priorities are also identical. In this case, we denote $c_{0} \underset{i}{\subset} c_{1}$. This mean that $c_{0}$ is included in $c_{1}$ at the index $i$. Then we have the following properties [5]: 
(A) [Main algorithm of naive exhaustive cache simulation for a cache $(s, b, a)$ and a memory address $A]$

1) Memory address $A$ is partitioned into its $\operatorname{tag} t$, index $i$, and offset $o$. Calculate $S(c, i)$ by using $c$ and $i$.

2) Check whether the priority queue $S(c, i)$ contains the tag $t$ or not.

3) If $S(c, i)$ contains $t$ in Step 2), a cache hit occurs on $c$ for $A$ and update $S(c, i)$ according to the FIFO replacement policy.

4) If $S(c, i)$ does not contain $t$ in Step 2), a cache miss occurs on $c$ for $A$. Add $t$ into $S(c, i)$ and update it according to the FIFO replacement policy.

(B) [Super-Cache-set-group based cache configuration simulation algorithm]

1) Generate a single cache set group including all the cache sets in $S S(i)=\{S(c, i) \mid c \in$ $C(s, b)\}$ for each number of sets $s$, block size $b$, and cache set index $i$.

2) For each memory access $A$, initialize the block size $b$ to be $b_{0}$, the number $s$ of cache sets to be $s_{0}$, and associativity $a$ to be two.

3) If we can skip the block size $b$ by applying CRCB-2 [6], go to Step 10).

4) Based on CRCB-1 [6], if we have a cache hit for $A$ on $(s, b, 1)$, go to Step 9) since we can skip every cache configuration $\left(s^{\prime}, b, a^{\prime}\right)$ where $s^{\prime}=s, 2 s, \ldots, s_{m}$ and $a^{\prime}=$ $1,2, \ldots, a_{m}$.

$5)$ Let $Q$ in $(s, b, a)$ be a priority queue that we have to check. If we already know that we have a cache hit on $Q$, go to Step 7$)$.

6) Check whether we have a cache hit on $Q$.

If a cache hit occurs in $Q$, we always have a cache hit in $\left(s, b, a^{\prime}\right)$ for $a<a^{\prime}$ in the super cache set group including $Q$. We can skip cache hit/miss checks for them. If a cache miss occurs in $Q$, update $Q$ accordingly and partition the super cache set group into two groups if necessary.

7) $a \leftarrow a+1$. If the new $(s, b, a)$ is within the cache configuration range, go to Step 5).

8) $a \leftarrow 2$ and $s \leftarrow 2 s$.

If the new $(s, b, a)$ is within the cache configuration range, go to Step 4$)$.

9) $s \leftarrow s_{0}$ and $b \leftarrow 2 b$.

If the new $(s, b, a)$ is within the cache configuration range, go to Step 3$)$.

10) If there is a next memory access, go to Step 2). Otherwise, stop.

Fig. 1. (A) Main algorithm for naive exhaustive cache simulation and (B) our proposed super-cache-setgroup-based cache configuration simulation algorithm (SCSG algorithm).

Property 1. When tag is hit at $S\left(c_{1}, i\right)_{j}$ and $j<a_{0}$, we also have a cache hit on $c_{0}$ and $c_{0} \underset{i}{\subset} c_{1}$ still holds. When tag is hit at $S\left(c_{1}, i\right)_{j}$ and $a_{0} \leq j<a_{1}$, we have a cache miss on $c_{0}$ and we have $c_{0} \underset{i}{\not} c_{1}$.

Property 2. When we have a cache miss for tag on $c_{1}$, we also have a cache miss for tag on $c_{0}$ but $c_{0} \underset{i}{\subset} c_{1}$ still holds true.

We can skip priority queue checking and updating in $c_{0}$ by just checking and updating priority queues in $c_{1}$ if $c_{0} \underset{i}{\subset} c_{1}$.

Based on the above two properties, we proposed in [5] a cache set group $(C S G)$ as shown in Fig. 2(A), in which we only simulate the maximumassociativity priority queue and skip the others. 


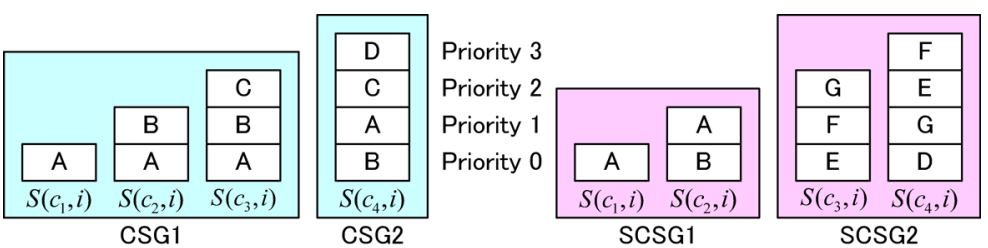

(A)

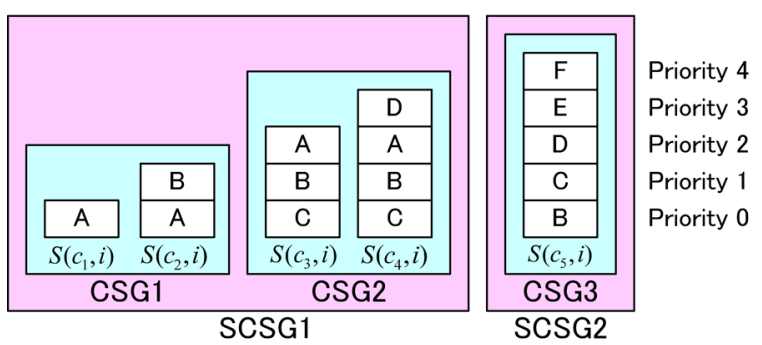

(B)

(C)

Fig. 2. (A) The priority queues $S\left(c_{1}, i\right), S\left(c_{2}, i\right)$, and $S\left(c_{3}, i\right)$ are in the cache-set-group CSG1. We can skip checking and updating of $S\left(c_{1}, i\right)$ and $S\left(c_{2}, i\right)$ by just checking and updating $S\left(c_{3}, i\right)$. CSG2 only includes a single priority queue. In CSG2, we skip no priority queues. (B) The proposed SCSG algorithm. Since both $S\left(c_{1}, i\right)$ and $S\left(c_{2}, i\right)$ are included in the same super cache set group, we can know that we have a cache hit on $S\left(c_{2}, i\right)$ without actually checking it if we have a cache hit on $S\left(c_{1}, i\right)$. Similarly, if we have a cache hit on $S\left(c_{3}, i\right)$, then we also have a cache hit on $S\left(c_{4}, i\right)$. (C) Combined CSG and SCSG algorithm.

As far as we know, the combined CRCB and CSG methods run the fastest for FIFO-based cache configuration simulation but we can further speed up them as described below.

\section{The proposed FIFO-based cache simulation speeding-up AI- gorithm - SCSG algorithm}

In this section, we will apply the property defined in Section 2.2 to FIFO relaxingly. Now let us focus on two cache configurations $c_{0}=\left(s, b, a_{0}\right)$ and $c_{1}=\left(s, b, a_{1}\right)$, where the number $s$ of cache sets is identical, their block size $b$ is also identical, but their associativity is different $\left(a_{0}<a_{1}\right)$. Let $S\left(c_{0}, i\right)$ and $S\left(c_{1}, i\right)$ be the $i$-th priority queues on $c_{0}$ and $c_{1}$, respectively. We assume here that all the tags in $S\left(c_{0}, i\right)$ are included in $S\left(c_{1}, i\right)$, but their priorities are not the same. In this case, we denote $c_{0} \underset{i}{\tilde{C}} c_{1}$. This means that $c_{0}$ is included relaxingly in $c_{1}$ at the index $i$. Even in this situation, we have the following properties for two cache configurations $c_{0}$ and $c_{1}$ such that $c_{0} \underset{i}{\tilde{C}} c_{1}$ :

Property 3. When a memory access tag $t$ is hit on $c_{0}$ at the index $i$, it is hit on $c_{1}$ and we can skip updating priority queues. In this case $c_{0} \underset{i}{\tilde{C}} c_{1}$ still holds. 
Proof. All the tags in the priority queue $S\left(c_{0}, i\right)$ are included in a priority queue $S\left(c_{1}, i\right)$. When $t$ is hit on $c_{0}$ at the index $i$, it is also included in $c_{1}$. Both priority queues $S\left(c_{0}, i\right)$ and $S\left(c_{1}, i\right)$ are not updated and $c_{0} \underset{i}{\tilde{C}} c_{1}$ still holds.

Property 4. When a memory access tag $t$ is missed on $c_{0}$ and hit on $c_{1}$ at the index $i, c_{0} \underset{i}{\tilde{C}} c_{1}$ still holds.

Proof. Before updating the priority queue $S\left(c_{0}, i\right), S\left(c_{1}, i\right)$ includes all the tags in $S\left(c_{0}, i\right)$, since $c_{0} \underset{i}{\tilde{C}} c_{1}$. When $t$ is missed on $c_{0}, t$ is inserted into $S\left(c_{0}, i\right)$. But, since $t$ is hit on $c_{1}$ at the index $i, S\left(c_{1}, i\right)$ already includes $t$. Then $c_{0} \underset{i}{\tilde{C}} c_{1}$ still holds after updating the priority queue $S\left(c_{0}, i\right)$.

Property 5. When a memory access tag $t$ is missed on both $c_{0}$ and $c_{1}$ at the index $i, c_{0} \underset{i}{\tilde{C}} c_{1}$ is not guaranteed to hold.

Proof. When $t$ is missed on $S\left(c_{1}, i\right)$, the largest-priority tag is kicked out of $S\left(c_{1}, i\right)$. Similarly, when $t$ is missed on $S\left(c_{0}, i\right)$, the largest-priority tag is kicked out of $S\left(c_{0}, i\right)$. When the largest-priority tag kicked out of $S\left(c_{1}, i\right)$ is included in $S\left(c_{0}, i\right), c_{0} \underset{i}{\tilde{C}} c_{1}$ no longer holds, that is, $c_{0} \underset{i}{\tilde{C}} c_{1}$ is not guaranteed to hold in this case.

Property 3 indicates that, when cache hit occurs on $c_{0}$ at the index $i$, we can skip priority queue checking and updating in $c_{1}$ if $c_{0} \underset{i}{\tilde{C}} c_{1}$ holds true.

Let $C(s, b)=\left\{(s, b, a) \mid s\right.$ and $b$ are fixed and $\left.a=1,2, \cdots, a_{m}\right\}$ be a set of cache configurations whose cache set size and block size are identical but their associativity is different. Let $S S(i)=\{S(c, i) \mid c \in C(s, b)\}$ be a set of cache sets whose index is $i$ in $C(s, b)$. $S S(i)$ is partitioned into several super cache set groups (SCSG) $S S(i)_{1}, S S(i)_{2}, \cdots$ as follows:

(1) For any cache configuration $c_{0} \in C(s, b)$, there exists a group which contains $S\left(c_{0}, i\right)$.

(2) For any two cache configurations $c_{0}, c_{1} \in C(s, b)$, if $c_{0} \underset{i}{\tilde{C}} c_{1}, S\left(c_{0}, i\right)$ and $S\left(c_{1}, i\right)$ are in the same group.

Fig. 2 (B) shows an example of super cache set groups. In each super cache set group, if we have a cache hit on a small-associativity priority queue, we also have cache hits on larger-associativity priority queues. We simulate the smaller-associativity priority queues one by one and may skip the largerassociativity priority queues.

Based on the above idea, we naturally propose a super-cache-set-group based algorithm (SCSG algorithm) as shown in Fig 1(B).

Furthermore, we can apply both the CSG algorithm [5] and the SCSG algorithm proposed above to FIFO. Fig. $2(\mathrm{C})$ shows the behavior of the combined CSG and SCSG algorithm. In Fig. 2(C), let us consider to check a cache hit/miss on SCSG1. We first check a cache hit on CSG1. If there exists a cache-hit priority queue in CSG1, we have cache hits on all the priority queues in CSG2 without really checking them. Further, when we 
Table I. Cache configuration simulation time comparisons.

\begin{tabular}{|c|c|c|c|c|c|c|}
\hline & \multicolumn{2}{|c|}{ DineroIV [2] } & \multicolumn{2}{|c|}{ CRCB [6] } & \multicolumn{2}{|c|}{$\mathrm{CRCB}+\mathrm{CSG}[5]$} \\
\hline & CPU time $[\mathrm{sec}]$ & Hit/miss check counts & CPU time [sec] & Hit/miss check counts & \begin{tabular}{l|l} 
its & CPU time [sec]
\end{tabular} & Hit/miss check counts \\
\hline ADPCME) & $244.9(30.5)$ & $631 \times 10^{6}$ & $8.04(1)$ & $65 \times 10^{6}$ & $7.63(0.95)$ & $31 \times 10^{6}$ \\
\hline ADPCM (D) & $251.7(31.0)$ & $608 \times 10^{6}$ & $8.11(1)$ & $67 \times 10^{6}$ & $7.69(0.95)$ & $32 \times 10^{6}$ \\
\hline JPEG (E) & $2223.2(62.3)$ & $30818 \times 10^{6}$ & $35.7(1)$ & $3285 \times 10^{6}$ & $27.2(0.76)$ & $2282 \times 10^{6}$ \\
\hline JPEG (D) & $546.2(33.7)$ & $3501 \times 10^{6}$ & $16.2(1)$ & $809 \times 10^{6}$ & $13.1(0.81)$ & $423 \times 10^{6}$ \\
\hline EPIC (E) & $3300.8(29.6)$ & $72963 \times 10^{6}$ & $111.6(1)$ & $11968 \times 10^{6}$ & $91.1(0.82)$ & $9762 \times 10^{6}$ \\
\hline $\operatorname{EPIC}(\mathrm{D})$ & $1011.6(20.5)$ & $12613 \times 10^{6}$ & $49.4(1)$ & $4428 \times 10^{6}$ & $34.1(0.69)$ & $3068 \times 10^{6}$ \\
\hline G721 (E) & $19415.9(360.9)$ & $136651 \times 10^{6}$ & $53.8(1)$ & $4078 \times 10^{6}$ & $65.8(1.22)$ & $1904 \times 10^{6}$ \\
\hline G721 (D) & $19921.6(415.0)$ & $139668 \times 10^{6}$ & $48.0(1)$ & $3115 \times 10^{6}$ & $40.2(0.84)$ & $1449 \times 10^{6}$ \\
\hline Total & 46915.9 (141.9) & $397457 \times 10$ & $330.6(1)$ & $27819 \times 10$ & $286.8(0.87)$ & $18955 \times 10^{6}$ \\
\hline & & \multicolumn{2}{|c|}{ CRCB + SCSG } & \multicolumn{2}{|c|}{ CRCB+CSG+SCSG } & \\
\hline & & CPU time $[\mathrm{sec}]$ & Hit/miss check counts & \begin{tabular}{l|l} 
its & CPU time [sec]
\end{tabular} & \multicolumn{2}{|c|}{ Hit/miss check counts } \\
\hline & ADPCME & $8.04(1.00)$ & $53 \times 10^{6}$ & $8.02(1.00)$ & \multicolumn{2}{|c|}{$31 \times 10^{6}$} \\
\hline & $\mathrm{ADPCM}(\mathrm{D})$ & $8.06(0.99)$ & $54 \times 10^{6}$ & $7.98(0.98)$ & \multicolumn{2}{|c|}{$31 \times 10^{6}$} \\
\hline & JPEG (E) & $25.5(0.71)$ & \multirow{2}{*}{$\begin{array}{r}1815 \times 10^{6} \\
376 \times 10^{6}\end{array}$} & $27.9(0.78)$ & \multicolumn{2}{|c|}{$2236 \times 10^{6}$} \\
\hline & JPEG (D) & $12.4(0.77)$ & & $12.9(0.80)$ & $415 \times 1$ & \\
\hline & EPIC (E) & $89.9(0.81)$ & $\begin{array}{r}376 \times 10^{6} \\
9606 \times 10^{6}\end{array}$ & $88.0(0.79)$ & $9205 \times 1$ & \\
\hline & $\operatorname{EPIC}(\mathrm{D})$ & $40.9(0.83)$ & $3894 \times 10$ & $35.1(0.71)$ & $3052 \times 1$ & \\
\hline & G721 (E) & $36.7(0.68)$ & $1014 \times 10$ & $42.0(0.78)$ & $1853 \times 1$ & \\
\hline & G721 (D) & $35.7(0.74)$ & $803 \times 10$ & $39.4(0.82)$ & $1362 \times 1$ & \\
\hline & Tota & $257.2(0.78)$ & $17619 \times 10$ & $261.3(0.79)$ & $18189 \times 1$ & \\
\hline
\end{tabular}

check if a cache hit occurs in CSG1 or not, we check a cache hit on $S\left(c_{2}, i\right)$ first. Based on Property 1, we may skip cache hit/miss check on $S\left(c_{1}, i\right)$.

\section{Experimental evaluations}

Through the experimental evaluations, we have obtained cache hit/miss counts for (i) Dinero IV [2], (ii) the CRCB method (CRCB), (iii) combined CRCB method and CSG method (CRCB+CSG), (iv) combined CRCB method and our proposed super-cache-set-group based algorithm (CRCB+SCSG), and (v) combined CRCB method, CSG method, and our proposed SCSG method (CRCB+CSG+SCSG). Dinero IV is a well-known standard cache simulator based on an exhaustive approach, which can be applied to FIFObased caches. All these cache simulation algorithms ran on Xeon $3.0 \mathrm{GHz}$ CPU and 4 GB memory PC. Application programs that we have used here are from MediaBench [4]. We have also used SimpleScalar [1] to obtain memory access histories. We have totally 925 cache configurations whose total cache size ranges from 256 bytes $(s=32, b=8$ bytes, and $a=1)$ to 134217728 bytes $(s=1024, b=1 \mathrm{~KB}$, and $a=128)$.

Table I summarizes the experimental results. Obtained cache hit/miss counts for every cache configuration were exactly the same for Dinero IV, $\mathrm{CRCB}, \mathrm{CRCB}+\mathrm{CSG}, \mathrm{CRCB}+\mathrm{SCSG}$, and CRCB+CSG+SCSG. Roughly saying, $\mathrm{CRCB}+\mathrm{SCSG}$ and $\mathrm{CRCB}+\mathrm{CSG}+\mathrm{SCSG}$ run on average $22 \%$ and $21 \%$ faster, respectively, than $\mathrm{CRCB}$. $\mathrm{CRCB}+\mathrm{SCSG}$ and $\mathrm{CRCB}+\mathrm{CSG}+\mathrm{SCSG}$ run on average $10 \%$ and $9 \%$ faster than $\mathrm{CRCB}+\mathrm{CSG}$, respectively. $\mathrm{CRCB}+$ SCSG runs $2 \%$ faster than CRCB+CSG+SCSG. This may be due to the overheads of combing CSG and SCSG. Overall, introducing the SCSG algorithm leads to the world fastest FIFO-based cache configuration simulation.

\section{Acknowledgement}

The research is supported in part by KAKENHI (No. 22300019), Waseda University Grant for Special Research Projects (No. 2011A-045), the Telecommunication Advancement Foundation, and the Kurata Memorial Hitachi Science and Technology Foundation. 